\section{Effects of triclosan on host response and microbial biomarkers during experimental gingivitis}

Pancer BA, Kott D, Sugai JV, Panagakos FS, Braun TM, Teles RP, Giannobile WV, Kinney JS. Effects of triclosan on host response and microbial biomarkers during experimental gingivitis. J Clin Periodontol 2016; 43: 435-444. doi: 10.1111/jcpe.12519.

\begin{abstract} curves and predict gingivitis susceptibility. $P$. gingivalis during induction of gingivitis. (ClinicalTrials.gov NCT01799226).
\end{abstract}

Aim: This exploratory randomized, controlled clinical trial sought to evaluate anti-inflammatory and -microbial effects of triclosan during experimental gingivitis as assessed by host response biomarkers and biofilm microbial pathogens. Materials and Methods: Thirty participants were randomized to triclosan or control dentifrice groups who ceased homecare for 21 days in an experimental gingivitis (EG) protocol. Plaque and gingival indices and saliva, plaque, and gingival crevicular fluid (GCF) were assessed/collected at days 0, 14, 21 and 35. Levels and proportions of 40 bacterial species from plaque samples were determined using checkerboard DNA-DNA hybridization. Ten biomarkers associated with inflammation, matrix degradation, and host protection were measured from GCF and saliva and analysed using a multiplex array. Participants were stratified as "high" or "low" responders based on gingival index and GCF biomarkers and bacterial biofilm were combined to generate receiver operating characteristic

Results: No differences in mean PI and GI values were observed between groups and non-significant trends of reduction of host response biomarkers with triclosan treatment. Triclosan significantly reduced levels of $A$. actinomycetemcomitans and

Conclusions: Triclosan reduced microbial levels during gingivitis development
Gingivitis is a reversible inflammatory periodontal disease associated with dental plaque. It appears in high prevalence, with estimations of more than half of the United States' adults. (Loe et al. 1978, Eke et al. 2012).

The classic model of experimental gingivitis (EG) was developed in 1965 by Löe et al. and is routinely used today to study the pathogenesis and microbiologic changes from
Brooke A. Pancer ${ }^{1}$, Diana Kott ${ }^{1}$, James V. Sugai ${ }^{1}$, Fotinos $\mathrm{S}$. Panagakos $^{2}$, Thomas M. Braun ${ }^{3}$, Ricardo P. Teles ${ }^{4,5}$, William V. Giannobile $^{1}$ and Janet S. Kinney ${ }^{1}$

${ }^{1}$ Department of Periodontics and Oral Medicine, Michigan Center for Oral Health Research, University of Michigan, Ann Arbor, MI, USA; ${ }^{2}$ Colgate-Palmolive Technology Center, Piscataway, NJ, USA; ${ }^{3}$ Biostatistics Department, School of Public Health, University of Michigan, Ann Arbor, MI, USA; ${ }^{4}$ Department of Periodontology, School of Dentistry, University of North Carolina, Chapel Hill, NC, USA; ${ }^{5}$ Department of Applied Oral Sciences, The Forsyth Institute, Cambridge, MA, USA

Key words: anti-inflammatory agents; bacteria; dental plaque/microbiology; dentifrices; gingivitis; inflammation mediators/analysis

Accepted for publication 19 January 2016 health to gingivitis (Loe et al. 1965). Most recently, this model has been adapted to examine changes in oral fluid inflammatory biomarkers (Lee et al. 2012). This study identified changes in 10 inflammatory biomarkers and 40 pathogens during induction and resolution of gingivitis (EG). They also stratified subjects based on their clinical inflammatory response and used baseline levels of salivary biomarkers to predict that 
response. Others have since confirmed these findings demonstrating changes in gingival crevicular fluid (GCF) biomarkers with induction of gingivitis using this model or the traditional EG model (Heasman et al. 1993, Offenbacher et al. 2007, 2010, Salvi et al. 2010, Trombelli et al. 2010, Farina et al. 2012, Scott et al. 2012, Eberhard et al. 2013).

Triclosan is a preservative and broad-spectrum anti-microbial agent (Jones et al. 2000). It has significant anti-inflammatory properties by inhibiting the cyclooxygenase and lipoxygenase pathways of arachidonic acid metabolism (Muller et al. 2006) as well as host-derived inflammatory mediators such as interleukin (IL) $1 \beta$, IL-6, tumour-necrosis factor, and prostaglandins (PG) (Modeer et al. 1996, Barros et al. 2010, Trombelli \& Farina 2013). Triclosan also significantly reduces plaque, calculus, and gingivitis, especially in patients who have pre-existing established gingivitis (Hioe \& van der Weijden 2005, Panagakos et al. 2005, Muller et al. 2006, Teles \& Teles 2009, Vered et al. 2009, Riley \& Lamont 2013). Additionally, when used in conjunction with tooth brushing, it has also been shown to reduce Fusobacteria and Veillonella (Arweiler et al. 2002, Fine et al. 2006) and to inhibit IL-1 $\beta$, IL6, and $\mathrm{PGE}_{2}$ (Modeer et al. 1996, Panagakos et al. 2005, Muller et al. 2006, Barros et al. 2010).

Stent models have been utilized to evaluate anti-inflammatory effects of triclosan dentifrice during EG. While Saxton and colleagues found superior reductions in plaque formation and gingivitis with triclosan as compared to a control (Saxton \& van der Ouderaa 1989), others were unable to demonstrate such clinical differences (Saxton et al. 1993, Lang et al. 2002). These studies, however, only focused on clinical parameters and contained dentifrice formulations that are not currently available.

The aim of this exploratory study was to assess the anti-inflammatory effects of triclosan dentifrice compared to a control during experimental gingivitis. The primary outcome was to examine GCF biomarker analytes. Periodontal clinical measurements, microbial analysis, and whole saliva biomarker analyses were also performed.

\section{Materials and Methods}

\section{Study population}

Participants provided written informed consent to the protocol approved by the University of Michigan Health Sciences Institutional Review Board before administration of research-related procedures. The investigation was conducted at the Michigan Center for Oral Health Research in accordance with the Helsinki Declaration of 1975, as revised in 2000. The inclusion and exclusion criteria of the study population are outlined in Appendix S1.

\section{Clinical procedures}

Figure 1 summarizes the study timeline. A double-blind, controlled, parallel group study modelling the EG protocol originally described by Löe and Theilade was performed (Loe et al. 1965). At screening (day -14) a periodontal evaluation, dental prophylaxis, and oral hygiene instruction were performed $(n=78)$. Fullmouth $\mathrm{PD}$, recession (REC), CAL, and BOP were recorded using a periodontal probe at six sites per tooth on all teeth except the third molars. Participants provided a urine specimen to confirm their non-smoking status. Pregnancy status was obtained through questionnaires given at each study visit. Mandibular alginate impressions were taken and customized stents were made by a single examiner based on a modification of a previously described study (Saxton \& van der Ouderaa 1989). Appendix S2 provides a description of the stent fabrication. At day 0 (baseline), if all inclusion and exclusion criteria were met, participants entered the experimental phase of the trial. Participants who met all inclusion criteria but had a BOP score of $>10 \%$ were given additional oral hygiene instruction and returned in 2 weeks for a second assessment. Participants not reaching a BOP score of $\leq 10 \%$ by the second confirmation visit were not enrolled in the study.

Using an online randomization chart, participants were assigned to either the test (triclosan dentifrice) or control (fluoride dentifrice) arm of the study as well as to either the right or left stent side. During the induction phase (i.e., baseline to 21 days), participants were instructed to refrain from all hygiene procedures in the stent area. During this time period, participants delivered a total of $2 \mathrm{ml}$ of their assigned dentifrice into their stent, allowing it to come into contact with the areas of EG for $2 \mathrm{~min}$. twice daily while traditional tooth brushing was performed in the non-stent areas. A complete description of participant homecare instructions can be found in Appendix S3.

The test dentifrice was composed of $0.24 \%$ sodium fluoride $1100 \mathrm{ppm}$ $0.243 \% \quad(0.14 \% \quad \mathrm{w} / \mathrm{v}$ fluoride ion $)$ and triclosan $0.30 \%$ in combination with $2 \%$ polyvinyl methyl ether maleic acid copolymer as the active ingredients along with inactive ingredients (Colgate ${ }^{\circledR}$ Total ${ }^{\circledR}$ Clean Mint Paste, Piscataway, NJ, USA). The control dentifrice was composed of $0.76 \%$ sodium monofluorophosphate $1000 \mathrm{ppm}(0.15 \% \mathrm{w} / \mathrm{v}$ fluoride ion) as the active ingredient along with inactive ingredients (Colgate ${ }^{\circledR}$ Cavity Protection Great Regular Flavor Fluoride Toothpaste). Both dentifrice types were packaged into identical white laminated tubes so as to ensure appropriate masking.

At 21 days, participants received a dental prophylaxis and reinstatement of homecare oral hygiene procedures from 21 to 35 days. All study materials were collected and compliance was assessed by calculating the amount of dentifrice used (Almerich et al. 2005). Re-establishment of gingival health was confirmed at day 35 .

At days $0,14,21$, and 35, the following data were collected in this sequence: whole saliva, intra-oral photos, GI, PI, plaque samples, and GCF. Gingival index (GI) (Loe \& Silness 1963) and plaque index (PI) (Silness \& Loe 1964) were taken from the stent quadrant Plaque and GCF samples were collected from the stent quadrant using a randomization chart to ensure no site was sampled twice. Repeat GCF samples were collected at $1,2,4$, and $6 \mathrm{~h}$ post-brushing at 14 and 21 days.

Five examiners underwent training and calibration procedures for PD, REC, CAL, BOP, PI, and GI prior to study initiation. Agreement was reached within $1 \mathrm{~mm}$ or better 

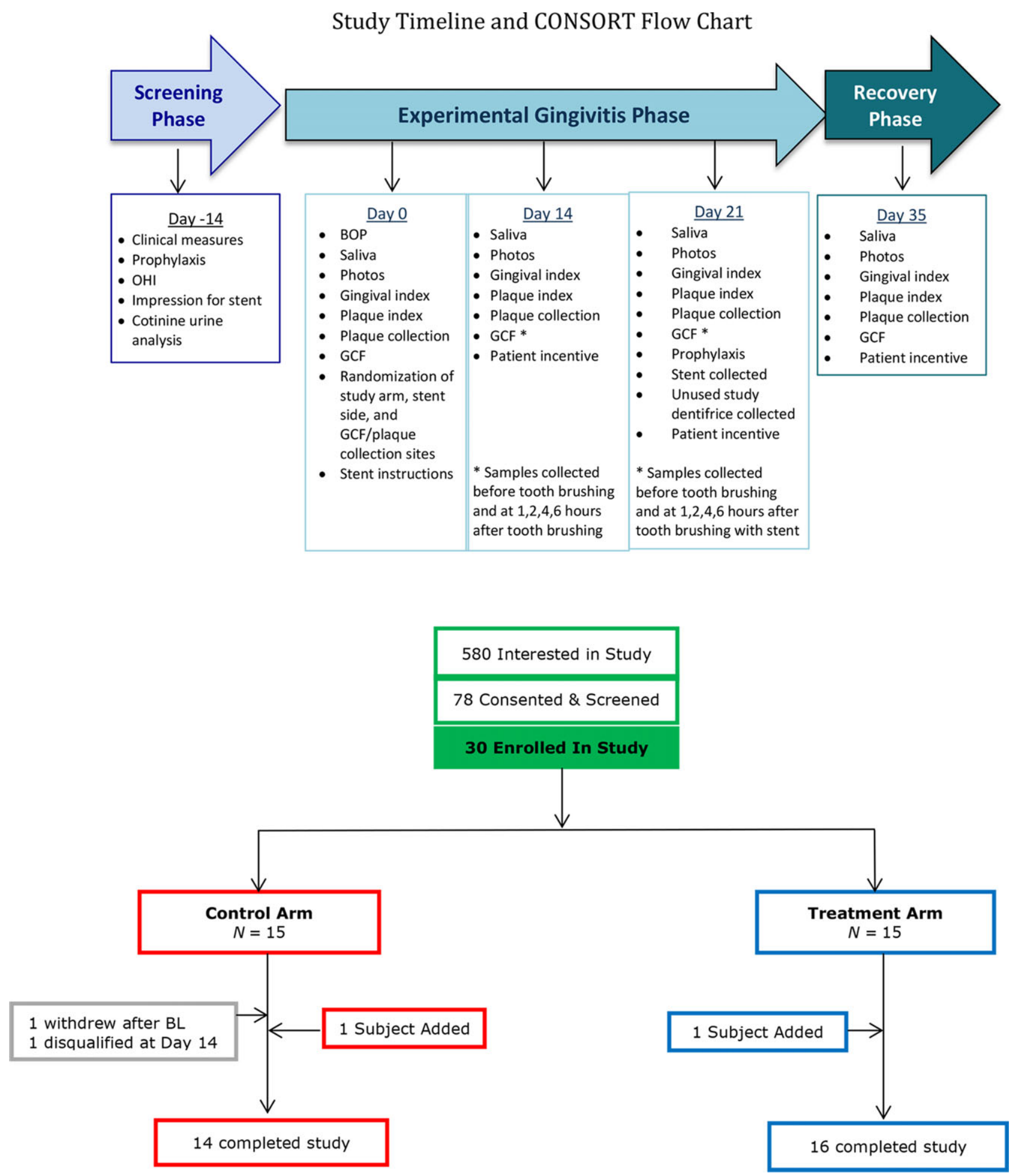

Fig. 1. Study timeline and recruitment and enrolment results of the study.

for measures of PD, REC, and CAL. For CAL measures, the mean inter-examiner correlation coefficient (using SPSS, Armonk, NY, USA) was 0.478 and the mean intra-examiner correlation coefficient (using SPSS) was 0.648. For PI and GI, rather a pre-study visual and tactile examiner training was performed. During the study, every attempt was made to partner the examiner with the same patient. Both participants and examiners were masked to the randomization scheme.

\section{Whole saliva collection and analysis}

Unstimulated whole saliva was collected at the beginning of each visit and processed as previously described (Kinney et al. 2011). Inflammatory biomarkers were 
quantified using a custom human 10complex protein array that was optimized for sensitivity, specificity, stability, and intra-assay coefficient of variation by comparing to single cytokine enzyme-linked immunosorbent assays (Quantibody Custom Array, RayBiotech, Norcross, GA). Based on the results from Lee and colleagues, the 10-biomarker panel consisted of: IL- $1 \alpha$, IL- $1 \beta$, IL-6, IL- 8 , IL10 , monocyte chemoattractant protein (MCP)-1, MMP-8, MMP-9, TIMP-1, and TIMP-2 (Lee et al. 2012). Appendix S4 provides additional information of the array procedures. Immediately after whole saliva collection, intra-oral photos were taken.

\section{Microbial plaque collection and analysis}

Supra- and sub-gingival plaque samples were collected using a sterile curette from two sites within the stent area. A randomization chart ensured that each site in the quadrant was sampled only once. Samples were placed into vials containing $150 \mu \mathrm{l}$ of Tris-EDTA buffer $(10 \mathrm{mM}$ Tris-HCl, $1 \mathrm{mM}$ EDTA, and $500 \mathrm{ml}$ distilled water [pH 7.6]). $100 \mu \mathrm{l}$ of $0.5 \mathrm{M} \mathrm{NaOH}$ was added into each vial. Samples were stored at $4^{\circ} \mathrm{C}$ until processed. Appendix S5 provides additional information regarding microbial analysis. Detection of 40 bacterial species was evaluated by the checkerboard DNA-DNA hybridization technique originally described by Socransky \& Haffajee 1994, (Socransky \& Haffajee 1994, Lee et al. 2012). The species evaluated and their corresponding microbial complexes are listed in Fig. $S 1$.

\section{GCF collection and analysis}

Gingival crevicular fluid samples were collected at all study time points from the same two sites as the microbial plaque samples were taken. At 14 and 21 days, GCF samples were collected, then patients were instructed to place the dentifrice in the stent and brush the rest of their teeth for a $2 \mathrm{~min}$. unsupervised session. Repeat GCF samples were collected from the same sites at 1, 2, 4, and $6 \mathrm{~h}$ post-brushing. A methylcellulose strip (Pro Flow, Inc., Amityville, NY, USA) was kept in place for $30 \mathrm{~s}$ and GCF was col- lected as previously described (Giannobile et al. 1995, Lamster \& Ahlo 2007). Following collection, strips were kept on dry ice for transport and then stored at $-80^{\circ} \mathrm{C}$ until analysis. The samples were thawed and proteins were extracted by an elution method (Giannobile et al. 1995). A complete description of the analysis procedures can be found in Appendix S6. Ten biomarkers were analysed based on the results from Lee and colleagues: IL-1 $\alpha$, IL-1 $\beta$, IL-6, IL-8, IL-10, MCP1, MMP-8, MMP-9, TIMP-1, and TIMP-2 (Lee et al. 2012).

\section{Statistical analyses}

See Appendix S7 for Comprehensive Statistical Analyses performed in this exploratory investigation.

\section{Results}

Figure 1 shows the CONSORT flow chart of participant recruitment and enrolment. Thirty participants completed the study; 14 in the control arm (2 males and 12 females; mean age: $27.1 \pm 5.2$ years) and 16 in the test arm (7 males and 9 females; mean age $26.1 \pm 5.2$ years). Table 1 depicts clinical and demographic information of the patients. No significant differences were found between the two groups in terms of race, age, and clinical parameters $(p>0.05)$.

\section{Clinical changes with induction of gingivitis}

Participants presented with high BOP scores at the screening visit and

Table 1. Participant demographics and clinical measures

\begin{tabular}{lccr}
\hline Characteristics & Test (frequency) & Control (frequency) & $p$-value \\
\hline Gender & $16(100 \%)$ & $14(100 \%)$ & NSD $^{*}$ \\
Male & $7(43.8 \%)$ & $2(14.3 \%)$ & \\
Female & $9(56.3 \%)$ & $12(85.7 \%)$ & \\
Race & $16(100 \%)$ & $14(100 \%)$ & NSD $^{*}$ \\
African American & $1(6.3 \%)$ & $1(7.1 \%)$ & \\
Asian & $2(12.5 \%)$ & $2(14.3 \%)$ & \\
Caucasian & $11(68.8 \%)$ & $9(64.3 \%)$ & \\
Hispanic & $1(6.3 \%)$ & $1(7.1 \%)$ & \\
Other & $1(6.3 \%)$ & $1(7.1 \%)$ & $0.60^{\dagger}$ \\
Age, mean (SD) & $26.06(5.23)$ & $27.07(5.15)$ & $0.66^{\dagger}$ \\
BOP at day -14 (SD) & $0.47(0.11)$ & $0.49(0.13)$ & $0.62^{\dagger}$ \\
BOP at day 0 (SD) & $0.09(0.04)$ & $0.10(0.06)$ & $0.93^{\dagger}$ \\
CAL at day - 14, mean (SD) & $0.76(0.25)$ & $0.75(0.34)$ & \\
\hline
\end{tabular}

NSD, no significant difference.

*Fisher's exact test.

${ }^{\dagger}$ Independent samples $t$-test. significantly reduced BOP scores at the baseline visit $(p<0.001)$ with no significant differences between the two groups. During the induction phase, significant increases in PI and GI were observed in both groups $(p<0.001)$ (Fig. 2a,b). A statistically significant difference was detected between groups at 14 days whereby the test group had increased plaque accumulation as compared to the control group $(p=0.017)$. All clinical parameters returned to baseline values at day 35, with significant differences seen from 21 days to 35 days in both groups $(p<0.001)$.

\section{Microbial changes with induction of gingivitis}

Total bacterial counts and counts of each microbial complex, except for the grey complex, increased significantly from baseline to 21 days and decreased significantly from 21 to 35 days $(p<0.05)$ (Fig. 3a,b).

A. actinomycetemcomitans was statistically significantly reduced in the test group as compared to controls from baseline to 14 days $(p=0.013)$ and from baseline to 21 days $(p=0.038)$. Statistically significant differences were also noted for $P$. gingivalis, which was markedly depressed by the triclosan dentifrice from baseline to 14 days as compared to control $(p=0.006)$.

\section{GCF and salivary biomarker changes with} induction of gingivitis

Both groups demonstrated mirrored and paralleled trajectories (Fig. 4). 

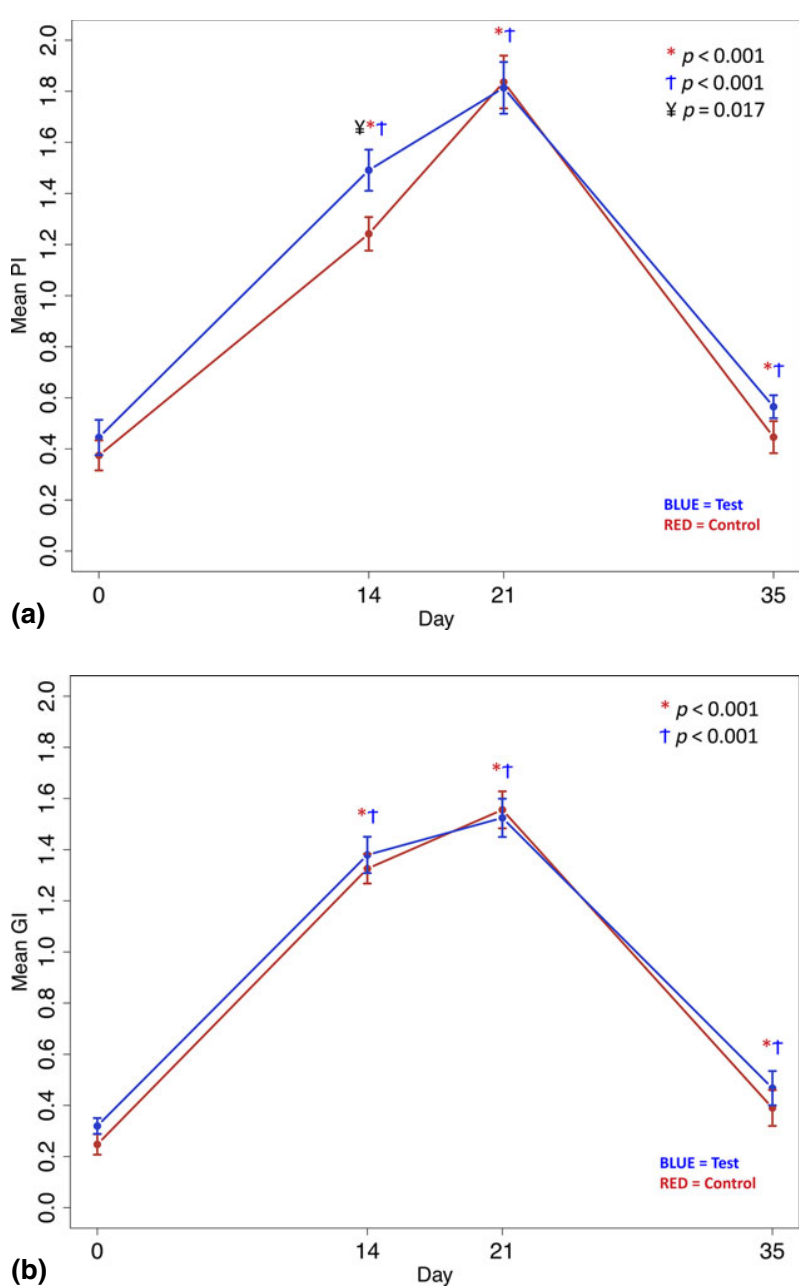

Fig. 2. A statistically significant increase in the plaque index and gingival index was noted with gingivitis induction as well as a statistically significant decrease with resolution $(p<0.001)$. At day 14 , the test group had a statistically significantly higher plaque index compared to the control group $\left({ }^{\ddagger} p=0.017\right)$.

GCF IL- $1 \alpha$ and IL- $1 \beta$ increased significantly from baseline to 21 days $(p<0.05)$ and decreased significantly during the recovery period for both groups. IL-8 and MCP-1 were significantly reduced during induction $(p<0.05)$ and increased during resolution $(p<0.05)$. TIMP-1 and TIMP-2 were significantly increased with resolution of gingivitis only in the test group $(p<0.05)$.

Though there were no significant differences at any hourly time points or areas under the curve (AUC) between the groups at 14 days or 21 days $(p>0.05)$ (Figs S2 and S3), several trends were noted.

No significant differences in salivary biomarkers were noticed over time or between dentifrice groups for any of the biomarkers (Fig. S4).

\section{Prediction of gingivitis response}

Sixteen participants belonged to the "high" responder group (9 test participants and 7 control), whereas 14 participants belonged to the "low" responder group (7 test participants and 7 control). IL- $1 \beta$ was the best single marker with an AUC of 0.64. The best pair combination was IL$1 \beta$ and MMP-8 with an AUC of 0.71 . The best trio combination was IL-1 $\beta$, MMP-8, and F. nucleatum ss polymorphum with an AUC of 0.85 with an odds ratio of $2.96(95 \% \mathrm{CI}=$ $1.16,7.57)$ of being a "high" responder (Fig. 5).

\section{Discussion}

The aims of this exploratory study were to assess how biomarkers and pathogens are modulated by triclosan dentifrice when used during induction of EG.

All patients developed gingivitis, with similar increases in GI and PI to previous studies (Loe et al. 1965, Offenbacher et al. 2010, Salvi et al. 2010, Lee et al. 2012). Our inability to demonstrate significant clinical differences between groups remains consistent with one study (Lang et al. 2002), yet contrasts with several other studies (Saxton et al. 1993). Vered et al. demonstrated remarkable effects of triclosan over control dentifrices when used for 6 months or longer (Vered et al. 2009), though a different study reported improved anti-gingivitis activity as early as 6 weeks (Panagakos et al. 2005). The underestimated actions of triclosan in our study may be related to limitations in our study design, specifically, our rigid inclusion criteria and small sample. As such, the effects of triclosan may not have been as dramatic as when used in a situation beginning with poorer oral hygiene, for example. It has been reported that participants with higher baseline gingival bleeding scores were more likely to show reductions in bleeding and inflammation using a triclosan dentifrice in a 3-month clinical trial (Shearer et al. 2005).

Interesting patterns of GCF biomarker results emerged, especially when comparing the study day $(0$, $14,21,35)$ longitudinal findings to repeat sampling at $0,1,2,4$ and $6 \mathrm{~h}$ post-dentifrice exposure. As expected, overall IL-1 $\beta$ levels increased in both groups during the induction phase of the study (day 0 to 21). Our results mirror those previously reported in other EG studies (Zhang et al. 2002, Trombelli et al. 2006, and Salvi et al. 2010). However, a marked drop in both IL-1 $\alpha$ and IL-1 $\beta$ concentrations was observed in both the test and controls groups at 14 days and 21 days $1 \mathrm{~h}$ post-dentifrice application. Furthermore, a continued sustained reduction in IL- $1 \alpha$ levels was seen for $6 \mathrm{~h}$ post exposure. Regarding IL8 production, our results are similar to others (Zhang et al. 2002, Deinzer et al. 2007, Lee et al. 2012) and showed a short-term down-regulation of this biomarker, especially early in the early stages of experimental gingivitis. What is notewor- 
Mean counts $(\times 105)$ of Microbial Species Stratified by Dentifrice Type

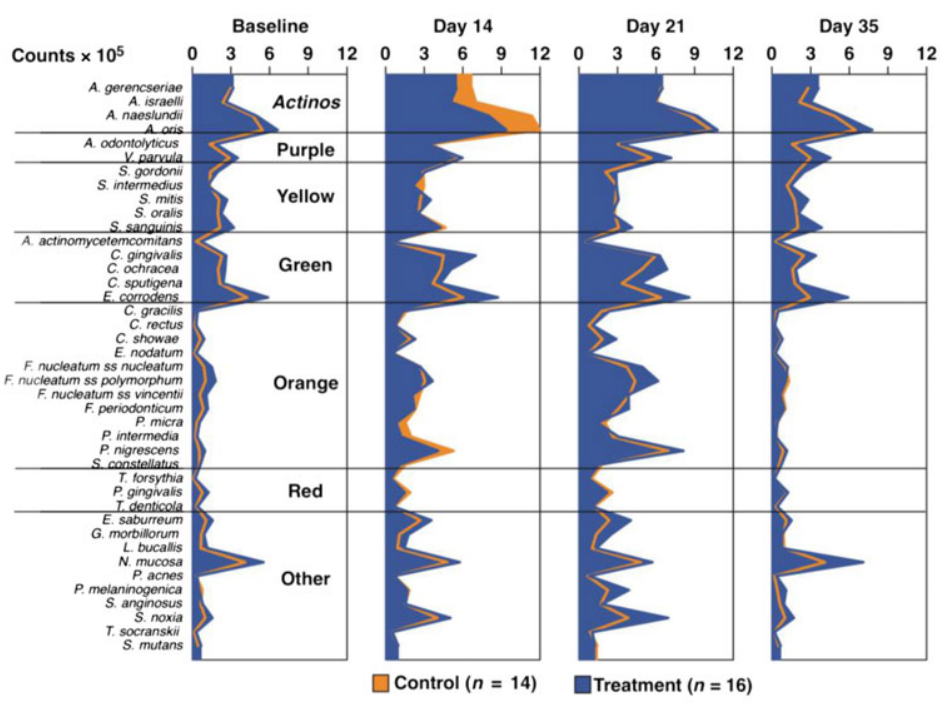

Pie Chart Demonstrating Progression and Proportions of Each Microbial Complex in Relation to Total DNA from Day 0 to Day 35 Stratified by Dentifrice Type

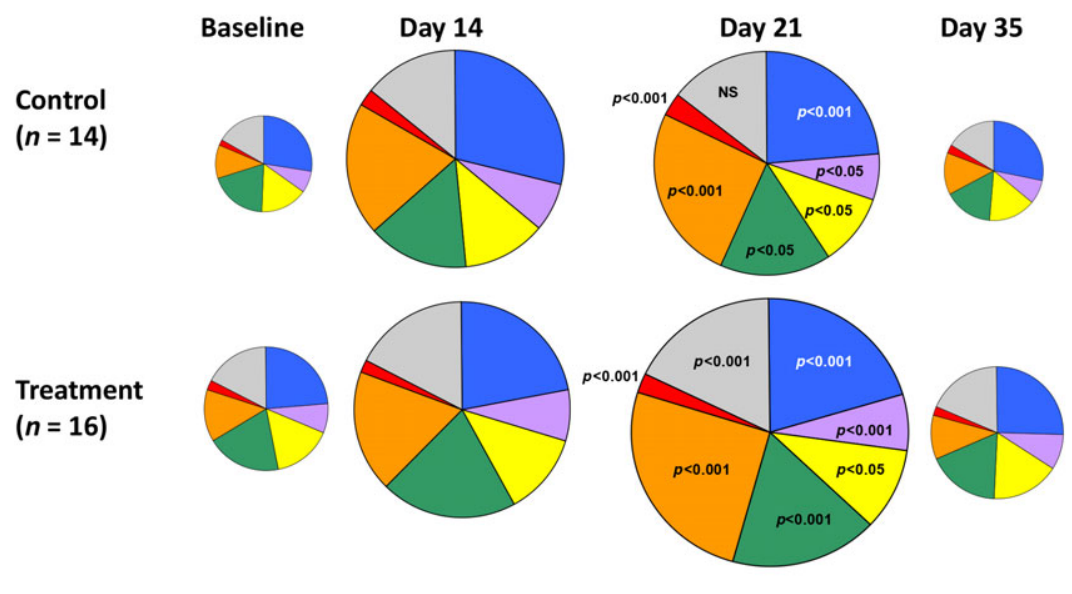

(b) P value calculated using Friedman test for changes over time.

Fig. 3. Blue line delineates bacterial counts in the test group. Orange fill represents bacterial counts in the control group.

thy is that our repeat IL-8 GCF sampling results at 14 days and 21 days showed a strong increase in this cytokine up to $2 \mathrm{~h}$ post-dentifrice application. After $2 \mathrm{~h}$ of dentifrice exposure levels of IL-8 appeared to plateau off, but were still elevated from pre-application concentrations. Other GCF biomarker differences were observed between the mean study day levels and the repeated sampling levels at 14 and 21 days. For example, while mean levels of MCP-1 decreased from day 0 to 14 and 21 days, postdentifrice contact levels 1, 2, 4 and
6 h steadily rose. A similar trend was seen in the mean levels of TIMP-2. There was an overall decrease in the concentrations of this biomarker during the induction phase of the study; yet repeat postdentifrice contact levels rose at 14 and 21 days.

The uniqueness of the model permitted evaluation of the effects of triclosan in the absence of tooth brushing and eliminated participant differences in oral hygiene practices as a potential bias. Furthermore, the model demonstrated differences in biomarker concentrations within hours after using the therapeutic agent. At 14 days, triclosan demonstrated trends of depressing levels of MMP-8 and -9 and TIMP-1 at 2 and $4 \mathrm{~h}$ after application through the stent. It was also able to maintain these depressed levels over the $6 \mathrm{~h}$ after application in MMP8 and TIMP-1. Furthermore, triclosan increased TIMP-2 levels just hours after its application on 21 days as demonstrated by the AUC analysis. This clearly demonstrates that triclosan has an immediate effect (within hours) in depressing levels of enzymes that destroy collagen in the early stages of gingivitis induction. In addition, after the depressed effects on the matrix degradation enzymes, triclosan was then able to increase the concentration of their inhibitors, further protecting the periodontium from destruction.

Based on our current knowledge, this is the first study demonstrating the anti-microbial effects of triclosan through DNA-DNA hybridization when used as a locally applied agent without the use of mechanical tooth brushing. The test group was found to have statistically significantly depressed changes in counts of Porphyromonas gingivalis and Aggregatibacter actinomycetemcomitans, as well as demonstrated trends for depressed levels of Tannerella forsythia. Changes in Actinomyces species were also found to be depressed, although not statistically significant, in the test as compared to the control. These species are commonly associated with developing gingivitis (Loesche \& Syed 1978, Slots 1979, Mombelli et al. 1990). The test group also demonstrated a trend for decreased levels of Peptostreptococcus micra and Fusobacterium nucleatum. P. micra has been shown to increase in gingivitis (Slots 1979, Mombelli et al. 1990) chronic periodontitis, and active progressive periodontal disease (Rams et al. 1992). Furthermore, its elimination has been associated with clinical improvement (Haffajee et al. 1988). $F$. nucleatum is a key component of the biofilm as it possesses the ability to coaggregate with multiple species in the oral cavity and bridge primary colonizers found in gingivitis with secondary colonizers such as $P$. gingivalis found in periodontitis (Yang et al. 2014). 

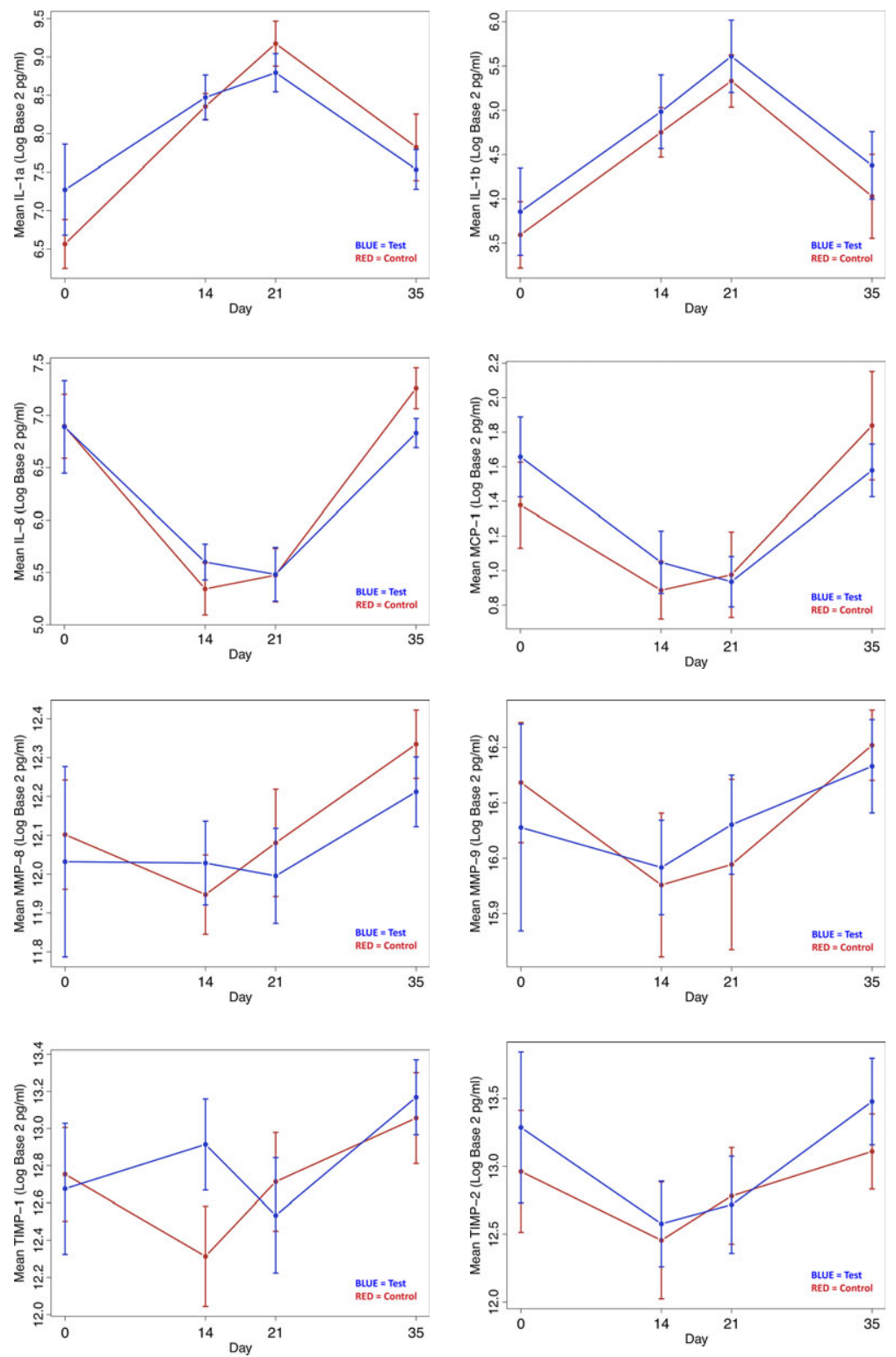

Fig. 4. GCF IL- $1 \alpha$ and IL- $1 \beta$ significantly increased with gingivitis induction (IL- $1 \alpha$; $\left.{ }^{*} p<0.001,{ }^{\dagger} p=0.005\right)\left(\mathrm{IL}-1 \beta ; * p<0.001,{ }^{\dagger} p=0.004\right)$ and decreased with resolution $\left(\right.$ IL- $\left.1 \alpha ; * p=0.006,{ }^{\dagger} p<0.001\right)($ IL- $1 \beta ; * p=0.020, \uparrow p=0.033)$. IL-8 and MCP-1 significantly decreased with gingivitis induction (IL-8; ${ }^{*} p<0.001,{ }^{\dagger} p=0.004$ ) (MCP-1; $\left.{ }^{*} p=0.046,{ }^{\dagger} p=0.004\right)$ and increased with resolution (IL-8; ${ }^{*} p<0.001,{ }^{\dagger} p<0.001$ ) $\left(\mathrm{MCP}-1 ;{ }^{*} p=0.039,{ }^{*} p=0.0 .03\right)$. No significant differences in matrix degradation enzymes were observed. TIMP-1 and TIMP-2 significantly increased with resolution of gingivitis in the test group (TIMP-1; $\left.{ }^{\dagger} p=0.020\right)\left(\right.$ TIMP- $\left.2 ;{ }^{\dagger} p=0.009\right)$.

Our findings remain consistent with former studies that demonstrate the benefit of triclosan dentifrice over a control when used in conjunction with tooth brushing (Arweiler et al. 2002, Fine et al. 2006). In this study we see declines in these microbial reductions, however, they were found to diminish considerably when meticulous patient home care and professional debridement was performed
(Teles \& Teles 2009). It is possible that trends and non-significant differences were noted in our study due to the small patient sample and extremely meticulous oral hygiene required by the participants prior to cessation of oral hygiene. Had the patients entered the experimental phase of the study with high levels of plaque and inflammation, it is possible that the effects of treatment may have been more noticeable. It is also possible that the anti-microbial and anti-inflammatory functions of triclosan may have been limited as a result of its placement and possible absorption into a thick, experimentally induced plaque filled surface. As such, we propose that the active chemical components, triclosan and fluoride, remain effective when used in conjunction with tooth brushing.

Baseline values of the combination of IL-1 $\beta$, MMP-8, and $F$. nucleatum ss polymorphum had the best ability to discriminate between "high" versus "low" responders. Doubling baseline values of IL- $1 \beta$ while controlling for MMP-8 and F. nucleatum ss polymorphum is associated with $3 \mathrm{x}$ the odds of displaying an exaggerated inflammatory response compared to those with low levels of at least one of these markers. MMP-8 was also shown to play an important role in predicting the inflammatory response. While our study did not demonstrate remarkable changes in this biomarker with gingivitis induction, others have shown an increase in MMP-8 with cessation of oral hygiene (Offenbacher et al. 2010, Salvi et al. 2010). Additionally, F.nucleatum as a bridging species has been found to predominate in gingivitis (Listgarten 1976, Slots 1979, Mombelli et al. 1990). In fact, Lee and colleagues also determined that this microbe predisposed participants to be at higher risk of gingivitis (Lee et al. 2012). Thus, "high" responders possess a specific microbial profile and immuno-inflammatory phenotype at baseline, which predicts colonization of periodontal pathogens and inflammatory cytokines.

Our exploratory study identified changes in host immune and microbial biomarkers that transpire during the pathogenesis of gingival inflammation. Though our pilot study was unable to demonstrate superior clinical effects, we did detect depressed levels in microbes and inflammatory GCF biomarkers during gingivitis development with the use of triclosan applied through a stent model. The test group demonstrated an increased ability to reduce Actinomyces species, red complex pathogens, A. actinomycetemcomitans, and bridging organisms $F$. nucleatum and $P$. micra with induction of gingivitis. 

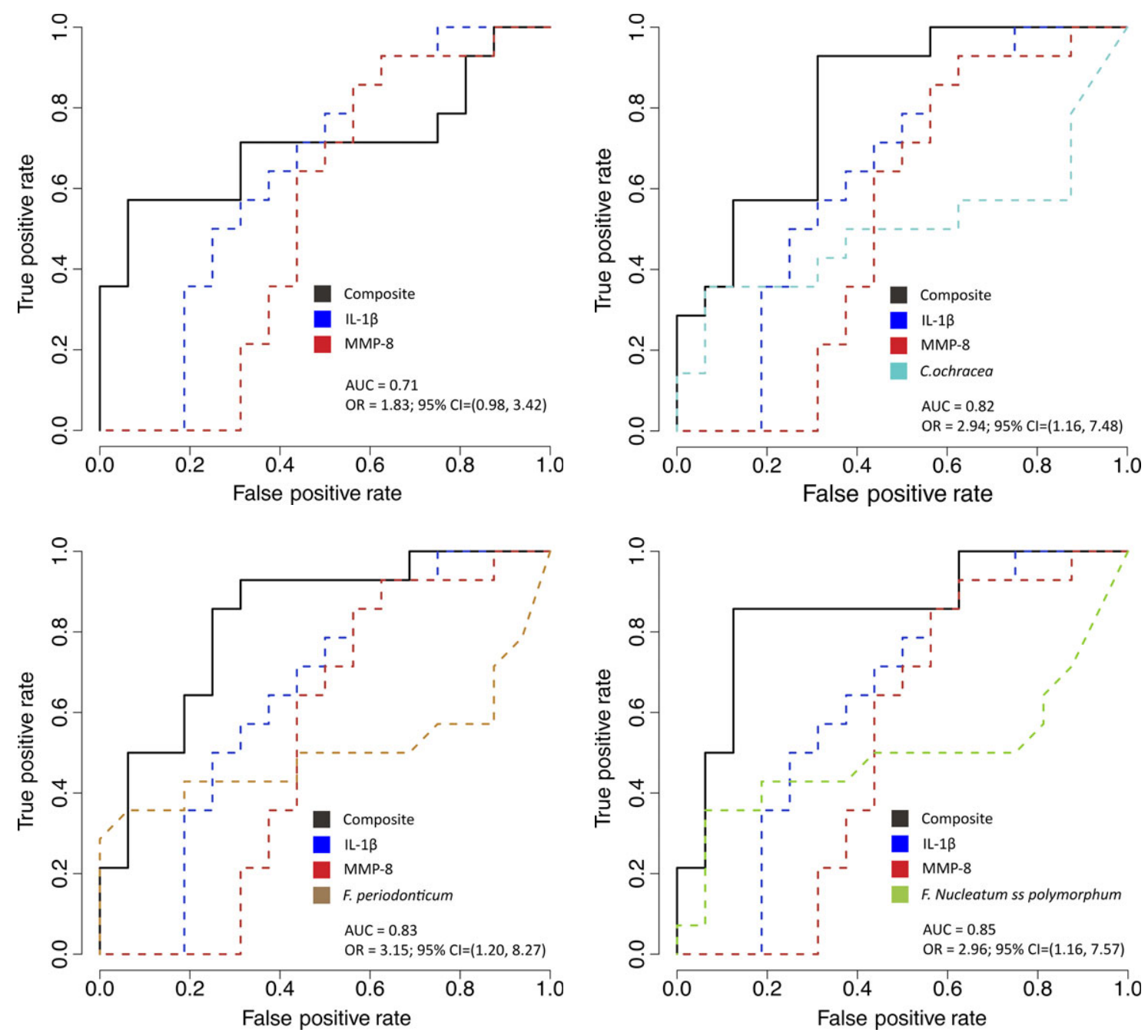

Fig. 5. The composite of IL-1 $\beta$, MMP-8, and F. nucleatum ss. Polymorphum generated an AUC of 0.85 . Doubling baseline values of IL-1 $\beta$ while controlling for MMP-8 and F. nucleatum ss polymorphum is associated with 2.96 times the odds of being a "high responder" compared to participants with low levels.

Triclosan also depressed levels of MMPs within a few hours after triclosan application early on in gingivitis development along with an increase in protective inhibitors of matrix degradation enzymes at the later stages of gingivitis development. By identifying highly susceptible patients, early identification, diagnosis, and therapeutic intervention may be provided prior to irreversible attachment loss. Given the nature of this exploratory trial, future larger randomized controlled clinical trials should be directed towards investigating a cluster of biomarkers affected by gingivitis along with therapeutic modalities that best reduce their immunoinflammatory and microbial component.

\section{Acknowledgements}

This work was supported by Colgate-Palmolive Company (Piscataway, NJ) and the National Institute of Health. Amy Collins and Erin Heffez (Giannobile Lab, University of Michigan, School of Dentistry, Ann Arbor, Michigan), Janet Riggs, Sara Wesley, Tina Ziba, Tina Huffman, Hily'e Pittman, Elizabeth Easter, and Anna Galloro (Michigan Center for Oral Health Research)

\section{Conflict of Interest}

Dr. William V. Giannobile has served as a consultant to Colgate, however not for this specific study. Dr. Foti Panagakos currently serves as Worldwide Director of Research Relations and Scientific Affairs, Colgate-Palmolive.

\section{References}

Almerich, J. M., Cabedo, B., Ortola, J. C. \& Poblet, J. (2005) Influence of alcohol in mouthwashes containing triclosan and zinc: an experimental gingivitis study. Journal of Clinical Periodontology 32, 539-544.

Arweiler, N. B., Auschill, T. M., Reich, E. \& Netuschil, L. (2002) Substantivity of toothpaste slurries and their effect on reestablishment of the dental biofilm. Journal of Clinical Periodontology 29, 615-621.

Barros, S. P., Wirojchanasak, S., Barrow, D. A., Panagakos, F. S., Devizio, W. \& Offenbacher, S. (2010) Triclosan inhibition of acute and chronic inflammatory gene pathways. Journal of Clinical Periodontology 37, 412-418.

Deinzer, R., Weik, U., Kolb-Bachofen, V. \& Herforth, A. (2007) Comparison of experimental gingivitis with persistent gingivitis: differences 
in clinical parameters and cytokine concentrations. Journal of Periodontal Research 42, 318 324.

Eberhard, J., Grote, K., Luchtefeld, M., Heuer, W., Schuett, H., Divchev, D., Scherer, R., Schmitz-Streit, R., Langfeldt, D., Stumpp, N., Staufenbiel, I., Schieffer, B. \& Stiesch, M (2013) Experimental gingivitis induces systemic inflammatory markers in young healthy individuals: a single-subject interventional study. PLoS One 8, e55265.

Eke, P. I., Dye, B. A., Wei, L., Thornton-Evans, G. O. \& Genco, R. J. \& CDC Periodontal Disease Surveillance Workgroup: James Beck, G. D. R. P. 2012. Prevalence of periodontitis in adults in the United States: 2009 and 2010. Journal of Dental Research, 91, 914-920.

Farina, R., Guarnelli, M. E., Figuero, E., Herrera, D., Sanz, M. \& Trombelli, L. (2012) Microbiological profile and calprotectin expression in naturally occurring and experimentally induced gingivitis. Clinical Oral Investigations, 16(5), 1475-1484.

Fine, D. H., Furgang, D., Markowitz, K., Sreenivasan, P. K., Klimpel, K. \& de Vizio, W. (2006) The antimicrobial effect of a triclosan/ copolymer dentifrice on oral microorganisms in vivo. Journal of the American Dental Association 137, 1406-1413.

Giannobile, W. V., Lynch, S. E., Denmark, R. G., Paquette, D. W., Fiorellini, J. P. \& Williams, R. C. (1995) Crevicular fluid osteocalcin and pyridinoline cross-linked carboxyterminal telopeptide of type I collagen (ICTP) as markers of rapid bone turnover in periodontitis. A pilot study in beagle dogs. Journal of Clinical Periodontology 22, 903-910.

Haffajee, A. D., Dzink, J. L. \& Socransky, S. S. (1988) Effect of modified Widman flap surgery and systemic tetracycline on the subgingival microbiota of periodontal lesions. Journal of Clinical Periodontology 15, 255-262.

Heasman, P. A., Collins, J. G. \& Offenbacher, S. (1993) Changes in crevicular fluid levels of interleukin-1 beta, leukotriene B4, prostaglandin E2, thromboxane B2 and tumour necrosis factor alpha in experimental gingivitis in humans. Journal of Periodontal Research 28, 241-247.

Hioe, K. P. \& van der Weijden, G. A. (2005) The effectiveness of self-performed mechanical plaque control with triclosan containing dentrifices. International Federation of Dental Hygienists 3 (4), 192-204.

Jones, R. D., Jampani, H. B., Newman, J. L. \& Lee, A. S. (2000) Triclosan: a review of effectiveness and safety in health care settings. American Journal of Infection Control 28, 184-196.

Kinney, J. S., Morelli, T., Braun, T., Ramseier, C. A., Herr, A. E., Sugai, J. V., Shelburne, C. E., Rayburn, L. A., Singh, A. K. \& Giannobile, W. V. (2011) Saliva/pathogen biomarker signatures and periodontal disease progression. Journal of Dental Research 90, 752-758.

Lamster, I. B. \& Ahlo, J. K. (2007) Analysis of gingival crevicular fluid as applied to the diagnosis of oral and systemic diseases. Annals of the New York Academy of Sciences 1098, 216229.

Lang, N. P., Sander, L., Barlow, A., Brennan, K., White, D. J., Bacca, L., Bartizek, R. D. \& McClanahan, S. F. (2002) Experimental gingivitis studies: effects of triclosan and triclosancontaining dentifrices on dental plaque and gingivitis in three-week randomized controlled clinical trials. Journal of Clinical Dentistry $\mathbf{1 3}$ 158-166.
Lee, A., Ghaname, C. B., Braun, T. M., Sugai, J. V., Teles, R. P., Loesche, W. J., Kornman, K S., Giannobile, W. V. \& Kinney, J. S. (2012) Bacterial and salivary biomarkers predict the gingival inflammatory profile. Journal of Periodontology 83, 79-89.

Listgarten, M. A. (1976) Structure of the microbial flora associated with periodontal health and disease in man. A light and electron microscopic study. Journal of Periodontology 47, 118.

Loe, H., Anerud, A., Boysen, H. \& Smith, M. (1978) The natural history of periodontal disease in man. The rate of periodontal destruction before 40 years of age. Journal of Periodontology 49, 607-620.

Loe, H. \& Silness, J. (1963) Periodontal disease in pregnancy. I. Prevalence and severity. Acta Odontologica Scandinavica 21, 533-551.

Loe, H., Theilade, E. \& Jensen, S. B. (1965) Experimental gingivitis in man. Journal of Periodontology 36, 177-187.

Loesche, W. J. \& Syed, S. A. (1978) Bacteriology of human experimental gingivitis: effect of plaque and gingivitis score. Infection and Immunity 21, 830-839.

Modeer, T., Bengtsson, A. \& Rolla, G. (1996) Triclosan reduces prostaglandin biosynthesis in human gingival fibroblasts challenged with interleukin-1 in vitro. Journal of Clinical Periodontology 23, 927-933.

Mombelli, A., Lang, N. P., Burgin, W. B. \& Gusberti, F. A. (1990) Microbial changes associated with the development of puberty gingivitis. Journal of Periodontal Research $\mathbf{2 5}$, 331-338.

Muller, H. P., Barrieshi-Nusair, K. M., Kononen, E. \& Yang, M. (2006) Effect of triclosan/ copolymer-containing toothpaste on the association between plaque and gingival bleeding: a randomized controlled clinical trial. Journal of Clinical Periodontology 33, 811-818.

Offenbacher, S., Barros, S., Mendoza, L., Mauriello, S., Preisser, J., Moss, K., de Jager, M. \& Aspiras, M. (2010) Changes in gingival crevicular fluid inflammatory mediator levels during the induction and resolution of experimental gingivitis in humans. Journal of Clinical Periodontology 37, 324-333.

Offenbacher, S., Barros, S. P., Singer, R. E. Moss, K., Williams, R. C. \& Beck, J. D. (2007) Periodontal disease at the biofilm-gingival interface. Journal of Periodontology 78, 19111925.

Panagakos, F. S., Volpe, A. R., Petrone, M. E., Devizio, W., Davies, R. M. \& Proskin, H. M. (2005) Advanced oral antibacterial/anti-inflammatory technology: a comprehensive review of the clinical benefits of a triclosan/copolymer fluoride dentifrice. The Journal of Clinical Dentistry 16 (Suppl), S1-S19.

Rams, T. E., Feik, D., Listgarten, M. A. \& Slots, J. (1992) Peptostreptococcus micros in human periodontitis. Oral Microbiology and Immunology 7, 1-6.

Riley, P. \& Lamont, T. 2013. Triclosan/copolymer containing toothpastes for oral health. Cochrane Database Systematic Review, 12, CD010514.

Salvi, G. E., Franco, L. M., Braun, T. M., Lee, A., Rutger Persson, G., Lang, N. P. \& Giannobile, W. V. 2010. Pro-inflammatory biomarkers during experimental gingivitis in patients with type 1 diabetes mellitus: a proof-of-concept study. Journal of Clinical Periodontology, 37, 9 16.
Saxton, C. A., Huntington, E. \& Cummins, D (1993) The effect of dentifrices containing triclosan on the development of gingivitis in a 21day experimental gingivitis study. International Dental Journal 43, 423-429.

Saxton, C. A. \& van der Ouderaa, F. J. (1989) The effect of a dentifrice containing zinc citrate and triclosan on developing gingivitis. Journal of Periodontal Research 24, 75-80.

Scott, A. E., Milward, M., Linden, G. J., Matthews, J. B., Carlile, M. J., Lundy, F. T., Naeeni, M. A., Lorraine Martin, S., Walker, B., Kinane, D., Brock, G. R. \& Chapple, I. L. (2012) Mapping biological to clinical phenotypes during the development (21 days) and resolution (21 days) of experimental gingivitis. Journal of Clinical Periodontology, 39 123-131.

Shearer, B., Hall, P., Clarke, P., Marshall, G. \& Kinane, D. F. (2005) Reducing variability and choosing ideal subjects for experimental gingivitis studies. Journal of Clinical Periodontology 32, 784-788.

Silness, J. \& Loe, H. (1964) Periodontal disease in pregnancy. II. Correlation between oral hygiene and periodontal condtion. Acta Odontologica Scandinavica 22, 121-135.

Slots, J. (1979) Subgingival microflora and periodontal disease. Journal of Clinical Periodontology 6, 351-382.

Socransky, S. S. \& Haffajee, A. D. (1994) Evidence of bacterial etiology: a historical perspective. Periodontology 20002000 (5), 7-25.

Teles, R. P. \& Teles, F. R. (2009) Antimicrobial agents used in the control of periodontal biofilms: effective adjuncts to mechanical plaque control? Brazilian Oral Research 23 (Suppl 1), $39-48$.

Trombelli, L., Scapoli, C., Calura, G. \& Tatakis, D. N. (2006) Time as a factor in the identification of subjects with different susceptibility to plaque-induced gingivitis. Journal of Clinical Periodontology 33, 324-328.

Trombelli, L. \& Farina, R. (2013) A review of factors influencing the incidence and severity of plaque-induced gingivitis. Minerva Stomatologica 62 (6), 207-234.

Trombelli, L., Scapoli, C., Carrieri, A., Giovanni, G., Calura, G. \& Farina, R. (2010) Interleukin1 beta levels in gingival crevicular fluid and serum under naturally occurring and experimental gingivitis. Journal of Clinical Periodontology 37 (8), 697-704

Vered, Y., Zini, A., Mann, J., Devizio, W., Stewart, B., Zhang, Y. P. \& Garcia, L. (2009) Comparison of a dentifrice containing $0.243 \%$ sodium fluoride, $0.3 \%$ triclosan, and $2.0 \%$ copolymer in a silica base, and a dentifrice containing $0.243 \%$ sodium fluoride in a silica base a three-year clinical trial of root caries and dental crowns among adults. The Journal of Clinical Dentistry 20, 62-65.

Yang, N. Y., Zhang, Q., Li, J. L., Yang, S. H \& Shi, Q. (2014) Progression of periodontal inflammation in adolescents is associated with increased number of Porphyromonas gingivalis, Prevotella intermedia, Tannerella forsythensis, and Fusobacterium nucleatum. International Journal of Paediatric Dentistry 24, 226-233.

Zhang, J., Kashket, S. \& Lingstrom, P. (2002) Evidence for the early onset of gingival inflammation following short-term plaque accumulation. Journal of Clinical Periodontology 29, 1082-1085. 


\section{Supporting Information}

Additional Supporting Information may be found in the online version of this article:

Fig. S1. 40 Microbial pathogens and their respective complexes analysed using the checkerboard DNA-DNA hybridization technique.

Fig. S2. No significant differences in pro-inflammatory cytokines were observed over time at day 14 .

Fig. S3. There was a trend for higher IL- $1 \beta$ values at the $1(p=0.087)$ and $2(p=0.076)$ hour time points in the

\section{Clinical Relevance}

Scientific rationale for the study: The effects of triclosan dentifrice on oral fluid biomarkers in the absence of mechanical plaque removal are unknown.

Principal findings: Local application of triclosan demonstrated an test group compared to the control at day 21 .

Fig. S4. No significant differences in salivary biomarkers were observed.

Table S1. Microbial changes during experimental gingivitis model.

Appendix S1. Patient inclusion and exclusion criteria.

Appendix S2. Description of stent fabrication.

Appendix S3. Participant instructions for using the stent.

Appendix S4. Supplemental information about salivary biomarker array procedures.

Appendix S5. Supplemental informa- tion about checkerboard DNA-DNA hybridization techniques.

Appendix S6. Supplemental GCF analysis information.

Appendix S7. Statistical analyses.

Address:

Janet Kinney

Division of Dental Hygiene

Department of Periodontics and Oral

Medicine

University of Michigan School of Dentistry

1011 N. University Ave

Ann Arbor 48109-1078

MI, USA

E-mail: kinneyj@umich.edu

ability to reduce microbial pathogens, of particular interest red complex pathogens and bridging organisms. It also lowered levels of matrix metalloproteinases early in the development of experimental gingivitis while increasing levels of protective inhibitors of collagen degradation enzymes later in the gingivitis state.

Practical implications: The use of a triclosan dentifrice may be considered a therapeutic intervention to alter the oral microbiome in patients with gingivitis. 